ORIGINAL ARTICLE

\title{
Angiopoietin switching regulates angiogenesis and progression of human hepatocellular carcinoma
}

\author{
K Sugimachi, S Tanaka, K Taguchi, S Aishima, M Shimada, K Sugimachi, M Tsuneyoshi
}

See end of article for authors' affiliations

......................

Correspondence to: Dr S Tanaka, Department of Surgery and Science, Graduate School of Medical Sciences, Kyushu University. 3-1-1

Maidashi, Higashi-ku Fukuoka 812-8582, Japan; shinjił@

surg2.med.kyushu-u.ac.jp

Accepted for publication 6 July 2003

\begin{abstract}
Aim: Angiopoietin 1 (Ang-1) and its antagonist, angiopoietin 2 (Ang-2), are novel ligands that regulate the Tie2 receptor. The Ang-2 gene is upregulated in the hypervascular type of human hepatocellular carcinoma (HCC). To gain a better understanding of the role of the Ang-Tie2 system in HCC the expression of these genes was investigated in a series of human HCCs.

Methods: The expression of the angiopoietin and Tie 2 proteins was investigated in nine normal liver tissues and 52 surgically resected HCCs. In addition, the effects of hypoxic stimuli on Ang-1, Ang-2, vascular endothelial growth factor (VEGF), and erythropoietin (EPO) expression was investigated in Hep3B cells. Results: Ang-1, rather than Ang-2, was more frequently expressed in the normal liver. Ang-1 was expressed in $68 \%$ of HCCs, whereas Ang-2 was expressed in $81 \%$, and was significantly higher in poorly differentiated HCCs characterised by high vascularity $(p=0.02)$, and in tumours with a peliotic change ( $p=0.02$ ). Strong expression of Tie 2 was seen in tumour vessels in accordance with Ang-2 expression. In Hep3B cells, hypoxic stimuli upregulated VEGF and EPO, but not Ang-1 or Ang-2.

Conclusions: These data support the evidence that the reversal of Ang-1 and Ang-2 expression plays an important role in the angiogenic and dedifferentiation processes in HCC. The hypoxic stimuli were not responsible for Ang-2 upregulation, unlike that of VEGF, in human HCC cells.
\end{abstract}

A ngiogenesis is required for a variety of physiological processes and also for continued tumour growth. ${ }^{1}$ Direct experimental evidence has shown that an avascular tumour rarely grows to a size larger than 2$3 \mathrm{~mm}^{2}$, but once a tumour becomes vascularised the progression of tumour growth and metastasis are rapid. ${ }^{12}$ The switch to the angiogenic phenotype involves many different factors and complex processes; both upregulation of angiogenic stimulators and downregulation of inhibitors. ${ }^{12}$

Hepatocellular carcinoma (HCC) is the third most common malignant tumour in Japan, and the prognosis is relatively poor, with progression being rapid. ${ }^{3}$ It has been generally accepted that HCC develops and progresses during dedifferentiation processes; from a small sized and well differentiated HCC with no developed blood vessels in the tumour to a larger sized and moderately or poorly differentiated HCC, with characteristic hypervascularity. ${ }^{5-7}$ We previously reported that angiopoietin 2 (Ang-2) mRNA was significantly overexpressed in hypervascular HCCs, and that the overexpression of Ang-2 resulted in rapid tumour growth and haemorrhage in an animal model system. ${ }^{8}$

Angiopoietins were originally identified as specific ligands of tyrosine kinase with immunoglobulin and epidermal growth factor homology domains 2 (Tie2)..$^{9}{ }^{10}$ Tie2 is a member of the endothelial cell specific receptor tyrosine kinase family, which is essential for the formation of the embryonic vasculature. ${ }^{11-13}$ Ang-1 is an agonistic ligand for Tie2, and Ang-2 is a naturally occurring antagonist that inhibits Ang-1 mediated Tie2 phosphorylation, at least under some circumstances. ${ }^{10}$ Angiopoietins and Tie2 are related to vascular remodelling and sprouting, which occur in a complementary and coordinated fashion during vascular development, along with vascular endothelial growth factor (VEGF) and its tyrosine kinase receptors (Flk-1 and Flt-1). ${ }^{14}$
"It has been generally accepted that HCC develops and progresses during dedifferentiation processes"

The upregulated expression of Ang- $2^{815-18}$ and Tie2 $2^{17} 20$ has been noted in some human malignant tumours, including HCC, and the important role of these factors in tumour angiogenesis has been discussed. Here, to clarify the dynamic role of the Ang-Tie2 system in HCC dedifferentiation and progression, we report the comprehensive expression patterns of the Ang-1, Ang-2, and Tie 2 proteins in a series of human normal liver and HCC tissues. We also examined angiopoietin mRNA expression in HCC cells under hypoxic conditions because the signal for Ang-2 upregulation in the tumour and the mechanisms involved have not been clearly identified. Tumour hypoxia, a powerful stimulus for angiogenesis, is linked to tumour progression and metastasis, ${ }^{21}$ and hypoxic stimulation of Ang-2 was reported in endothelial cells and glioma cells, ${ }^{22-24}$ although the regulation of Ang-2 in human HCC cells has not yet been well characterised.

The objective of our study was to examine the role and mechanisms of the Ang-Tie2 system in angiogenesis and the progression of HCC. The results obtained revealed a molecular switching mechanism, which may contribute to angiogenic processes involved in the progression of HCC.

\section{MATERIALS AND METHODS \\ Patients}

Our present retrospective study was based on data obtained using surgically resected tissues from 52 Japanese patients

Abbreviations: Ang, angiopoietin; EC, vascular endothelial cell; EPO, erythropoietin; HCC, hepatocellular carcinoma; HIF, hypoxia inducible factor; PC, vascular pericyte/smooth muscle cell; PCR, polymerase chain reaction; RT, reverse transcriptase; Tie2, tyrosine kinase with immunoglobulin and epidermal growth factor homology domains 2; VEGF, vascular endothelial growth factor 
who underwent hepatectomy for HCC at the department of surgery and science, Graduate School of Medicine of Kyushu University, Fukuoka, Japan from February 1995 to August 1998. Written informed consent was obtained from each patient before tissue acquisition. All the data were collected in the department of anatomic pathology, Graduate School of Medicine of Kyushu University. Fifty primary cases and two recurrent cases were included in our study. There were 41 men and 11 women, and their ages ranged from 45 to 83 years (mean, 63.5). All tumours were defined as HCC, and the pathological features of the tumours were evaluated histologically (by KS, KT, SA, and MT), based on the classification of the liver cancer study group of Japan. ${ }^{25}$ The histological grades of tumours that consisted of more than two features were defined by the prominent feature, and these components were selected for the immunohistochemical studies. There were six well differentiated HCCs, 34 moderately differentiated ones, and 12 poorly differentiated ones. Table 1 summarises the clinicopathological features of these 52 patients. We also investigated liver tissue with no histological abnormalities obtained from nine patients who underwent necropsy or hepatectomy related to non-liver associated disease or liver contusion, and these tissues served as a control of non-diseased normal liver tissue in our study.

\section{Immunohistochemical staining of Ang-1, Ang-2, Tie2, and CD3 1}

Formalin fixed, paraffin wax embedded tissue sections were processed for immunohistochemical staining. Endogenous peroxidase activity was blocked using methanol containing $0.3 \% \mathrm{H}_{2} \mathrm{O}_{2}$. For antigen retrieval, sections were incubated in trypsin (1 $\mu \mathrm{g} / \mathrm{ml})$ (Sigma Chemical, St Louis, Missouri, USA) at $37^{\circ} \mathrm{C}$ for 30 minutes. Non-specific antibody binding was blocked by incubation with $10 \%$ normal rabbit serum (Nichirei, Tokyo, Japan) for 20 minutes. Endogenous biotin was blocked using an endogenous avidin-biotin blocking kit (Nichirei).

Sections were incubated with primary polyclonal antibodies against Ang-1 (Santa Cruz Biotechnology, Santa Cruz, California, USA; diluted 1/150), ${ }^{26}$ Ang-2 (Santa Cruz; diluted 1/150), ${ }^{26}{ }^{27}$ Tie2 (Santa Cruz; diluted $\left.1 / 250\right),{ }^{27}$ and CD31 (Dako A/S, Glostrup, Denmark; diluted 1/20) overnight at $4^{\circ} \mathrm{C}$, and then the bound antibodies were visualised, using streptavidin-biotin-peroxidase kits (Histofine ${ }^{\circledR}$ SAB-PO kit; Nichirei) with diaminobenzidine tetrahydrochloride as the chromogen. Sections of human placenta served as positive controls for Ang-1, Ang-2, and Tie2. ${ }^{28}$ For the negative control, phosphate buffered saline was used instead of the primary antibody. Finally, the sections were lightly counterstained with haematoxylin.

\section{Evaluation of immunohistochemical staining}

Immunohistochemical staining was examined under a light microscope by two pathologists (KS and KT). The immunohistochemical expression patterns of Ang-1 and Ang-2 in HCC cells were classified as follows: those with $0 \%$ positive staining of carcinoma cells were graded as $(-)$; those between $1 \%$ and $10 \%$ as $(+)$; and those over $10 \%$ as $(2+)$. We selected $10 \%$ as the cut off value because we could clearly divide expression into sparse and localised expression or diffuse expression at the $10 \%$ value. To evaluate Tie 2 expression, blood vessels in the tumour expressing Tie 2 and CD31 were counted, as described by Peters et al, ${ }^{19}$ and sinusoid-like vessels that were positive for CD31 were excluded to perform a quantitative analysis. Using a low magnification field $(\times 40)$, the most highly vascularised areas of each tissue were identified by scanning the CD31 stained sections. The CD31 positive vessels were then counted in three different high magnification fields $(\times 200)$. Tie 2 positive vessels were counted in the same regions in which CD31 had been determined. The ratio of the Tie 2 to CD 31 counts was considered to represent the Tie 2 expression level in each specimen. ${ }^{19}$

\section{Cell culture and hypoxia conditions}

Hep3B cells, a cultured human HCC cell line, were cultured at $37^{\circ} \mathrm{C}$ with $5 \% \mathrm{CO}_{2}$ in RPMI 1640 medium (Gibco BRL, Rockville, Maryland, USA), containing 10\% heat inactivated fetal bovine serum (Gibco BRL). Twenty four hours before exposure to hypoxia, cells were trypsinised and plated on $3.5 \mathrm{~cm}$ culture dishes. Hypoxia was achieved by incubating cells in a personal multigas incubator (APMW-36; Astec, Fukuoka, Japan) maintained in humidified 5\% $\mathrm{CO}_{2}, 1 \% \mathrm{O}_{2}$, and $94 \% \mathrm{~N}_{2}$ for 16 and 24 hours. Control cells were incubated in normoxic conditions $\left(5 \% \mathrm{CO}_{2}, 21 \% \mathrm{O}_{2}\right.$, and $\left.74 \% \mathrm{~N}_{2}\right)$, followed by immediate RNA extraction.

\section{Amplification of Ang-1, Ang-2, VEGF, and erythropoietin using RT-PCR}

Total RNA was extracted using Trizol Reagent (Gibco BRL), according to the manufacturer's instructions. The reverse transcriptase (RT) reaction was performed with $5 \mu \mathrm{g}$ of RNA,

\begin{tabular}{|c|c|c|c|c|}
\hline & Well $(n=6)$ & Moderate $(n=34)$ & Poor $(n=12)$ & Overall $(n=52)$ \\
\hline Mean (SD) age in years & $61.5(6.8)$ & $64.2(8.9)$ & $61.6(7.2)$ & $63.3(8.3)$ \\
\hline Men/women & & $28 / 6$ & $10 / 2$ & $41 / 11$ \\
\hline Mean (SD) tumour size $(\mathrm{cm})$ & $1.9(0.8)$ & $4.1(2.2)$ & $5.1(3.3)$ & $4.1(2.5)$ \\
\hline \multicolumn{5}{|l|}{ Virus markers } \\
\hline $\mathrm{HBsAg}(+)$ & 1 & 7 & 3 & 11 \\
\hline HCVAb $(+)$ & 6 & 26 & 10 & 42 \\
\hline HBsAg $(+)$ and HCVAb (+) & 1 & 3 & 1 & 5 \\
\hline \multicolumn{5}{|l|}{ Non-cancerous liver tissue } \\
\hline Normal or mild fibrosis & 0 & 1 & 0 & 1 \\
\hline Fibrosis & 1 & 16 & 6 & 23 \\
\hline Precirrhosis & 0 & 8 & 3 & 11 \\
\hline Cirrhosis & 5 & 9 & 3 & 17 \\
\hline Capsule formation & 1 & 31 & 11 & 43 \\
\hline Capsular invasion & 0 & 29 & 11 & 40 \\
\hline Septum formation & 0 & 26 & 9 & 35 \\
\hline Portal vein invasion & 0 & 16 & 11 & 27 \\
\hline Intrahepatic metastasis & 0 & 15 & 7 & 22 \\
\hline
\end{tabular}


random hexamers, and Superscript II reverse transcriptase (Gibco BRL), according to the manufacturer's instructions. The expression of Ang-1 and Ang-2 RNA was determined by 30 cycles of RT polymerase chain reaction (PCR) using the following primers: Ang-1, ${ }^{8}$ '-AAATGGAAGGAAAACA CAAGGAA-3' and 5'-ATCTGCACAGTCTCTAAATGGT-3' (annealing at $51{ }^{\circ} \mathrm{C}$; product size, $266 \mathrm{bp}$ ); Ang- $2,{ }^{8} 5^{\prime}-$ GACGGCTGTGATGATAGAAATAGG-3' and 5'-GACTGTAGTTGGATGATGTGCTTG-3' (annealing at $53^{\circ} \mathrm{C}$; product size, $264 \mathrm{bp}$ ). The resected samples of human HCC were used as positive controls for Ang-1 and Ang-2. ${ }^{8}$ We next analysed the expression of VEGF and erythropoietin (EPO), because they are known to be upregulated by hypoxia in Hep3B cells. ${ }^{29}$ RTPCR analysis of VEGF and EPO was performed using the following primers: VEGF, 5'-CTGTGTGCCCCTGATGCGAT GC-3' and 5'-CCTCCGGACCCAAAGTGCTCTG-3' (annealing at $60^{\circ} \mathrm{C}$; product size, $615 \mathrm{bp}$ (VEGF189), $543 \mathrm{bp}$ (VEGF165), or $411 \mathrm{bp}$ (VEGF121)); EPO, 5'-CAAATGGGCGGTAGGC GTGTA-3' and 5'-CATATAGACAAACGCACACC-3' (annealing at $60.5^{\circ} \mathrm{C}$; product size, $566 \mathrm{bp}$ ). As a control, RT-PCR analysis of $\beta$ actin mRNA was performed using $5^{\prime}$ AGGCCAACCGCGAGAAGATGACC-3' and 5'-GAAGTCC AGGGCGACGTAGCAC- $3^{\prime}$ as the primers (annealing at $60^{\circ} \mathrm{C}$; product size, $332 \mathrm{bp}$ ). All PCR primers were selected to span introns to detect specific mRNA sequences. The PCR mixtures contained $1 \mu \mathrm{l}$ of template cDNA ( $50 \mathrm{ng} / \mu \mathrm{l}$ ), $2 \mu \mathrm{l}$ of $1 \times$ PCR buffer (Takara Shuzo, Shiga, Japan), $1.6 \mu \mathrm{l}$ of dNTP mixture (Takara Shuzo), $0.4 \mu \mathrm{M}$ of each primer, and $0.5 \mathrm{U}$ of Takara Taq (Takara Shuzo) in a final volume of $20 \mu \mathrm{l}$. The reaction was run in a thermal cycler $\left(\right.$ GeneAmp ${ }^{\mathrm{TM}}$ PCR System 9600; Perkin-Elmer, Norfolk, Connecticut, USA). The PCR products were examined electrophoretically in agarose gels stained with ethidium bromide. The intensities of the bands were measured using image analysis software (Scion Image beta 4.02; Scion Corporation, Frederick, Massachusetts, USA). The gene expression ratio (intensity of PCR products with hypoxia normalised by $\beta$ actin expression/intensity of PCR products with normoxia normalised by $\beta$ actin expression) in each case was calculated.

\section{Statistical analysis}

Comparisons between angiopoietin expression patterns and clinicopathological parameters, and between angiopoietin expression and histology of normal liver tissue and HCC, were evaluated using $\chi^{2}$ tests, and comparisons between blood vessel counts and other parameters were evaluated using the Student's $t$ test. A p value of less than 0.05 was considered to be significant.

\section{RESULTS \\ Immunohistochemical study of Ang-1, Ang-2, and Tie2}

Ang-l expression was prominent in non-cancerous normal liver tissue. Of the nine normal liver tissues, eight had Ang-1 positive hepatocytes, whereas only two showed weak Ang-2 expression in the hepatocytes (fig 1). Among the 52 HCCs, the expression of Ang-2 was higher than that of Ang-1; Ang2 was partially positive in 25 tumours and diffusely positive in 17 tumours, whereas Ang-1 was partially positive in 25 tumours and diffusely positive in 10 tumours (fig 2). Figure 3 shows the expression patterns of Ang-1 and Ang-2 in normal liver tissues and HCCs. In normal liver tissue, Ang- 1 expression was higher than that of Ang-2. In contrast, Ang1 expression was lower than that of Ang-2 expression in well differentiated HCCs (17\% versus $33 \%$, respectively). In moderately and poorly differentiated HCC, both Ang-1 and Ang-2 were significantly overexpressed (74\% versus $87 \%$, respectively), compared with well differentiated HCCs ( $\mathrm{p}=0.02$ and $\mathrm{p}<0.01$, respectively). Therefore, the switching of Ang-1 and Ang-2 expression occurred during the progression from normal liver tissue to HCC.

Table 2 summarises the relation between the Ang-2 expression pattern and the clinicopathological features. The Ang-2 expression pattern significantly correlated with the histological differentiation of HCC. Strong positive expression of Ang-2 was most frequently seen in poorly differentiated HCC, followed by moderately differentiated HCC, whereas none of the well differentiated HCCs showed strong expression (fig 2). The staining intensity for Ang-2 was stronger in the peripheral area and areas with lymphocytic infiltration of the tumour, rather than in central and non-infiltrated areas. In HCC, a peliosis hepatis-like change (peliotic change), which is defined by the presence of cystic blood filled spaces within a tumour, is frequent, ${ }^{30}$ and positive Ang-2 immunoreactivity correlated well with the presence of peliotic change $(\mathrm{p}=0.02)$.

Microvessel counting of Tie 2 and CD31 positive vessels was performed in three different areas in all 52 HCC samples.
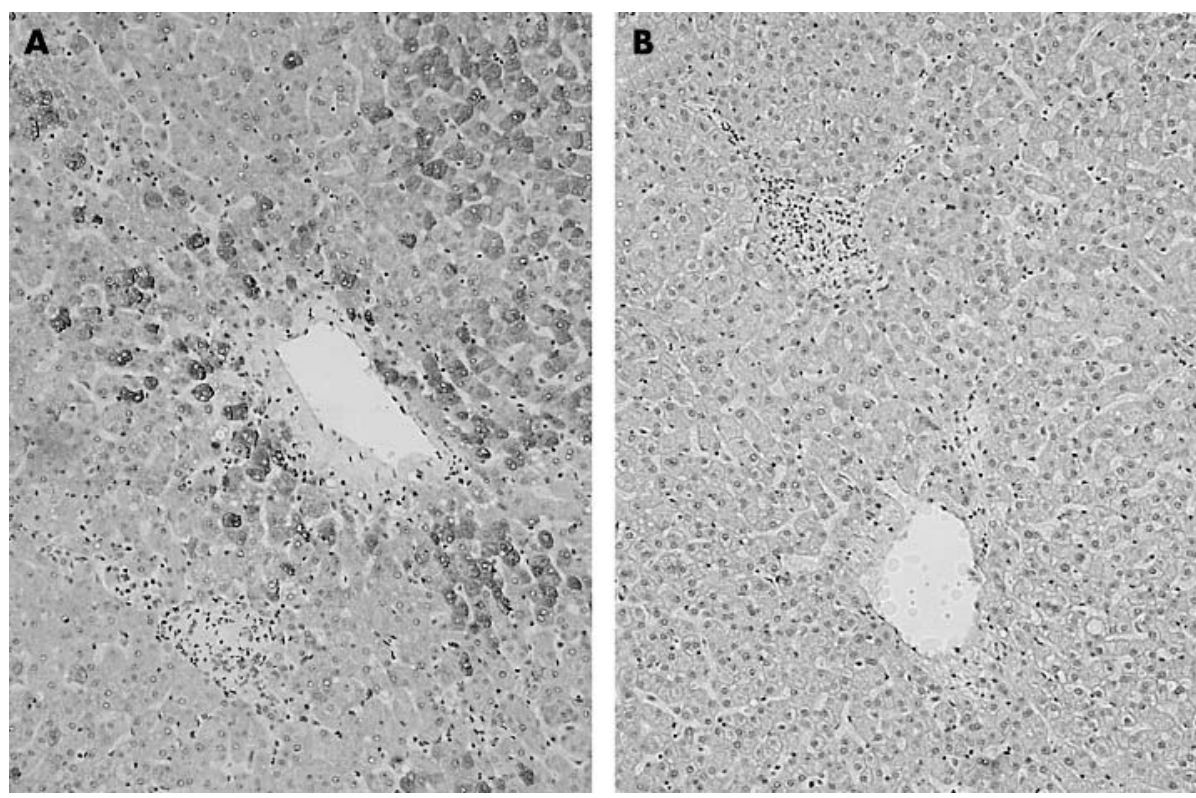

Figure 1 Immunohistochemistry of angiopoietins in normal human liver tissue. (A) Angiopoietin 1 (Ang-1) shows positive staining in normal hepatocytes. (B) Ang-2 is negative in normal hepatocytes. Original magnification, $\times 35$. 

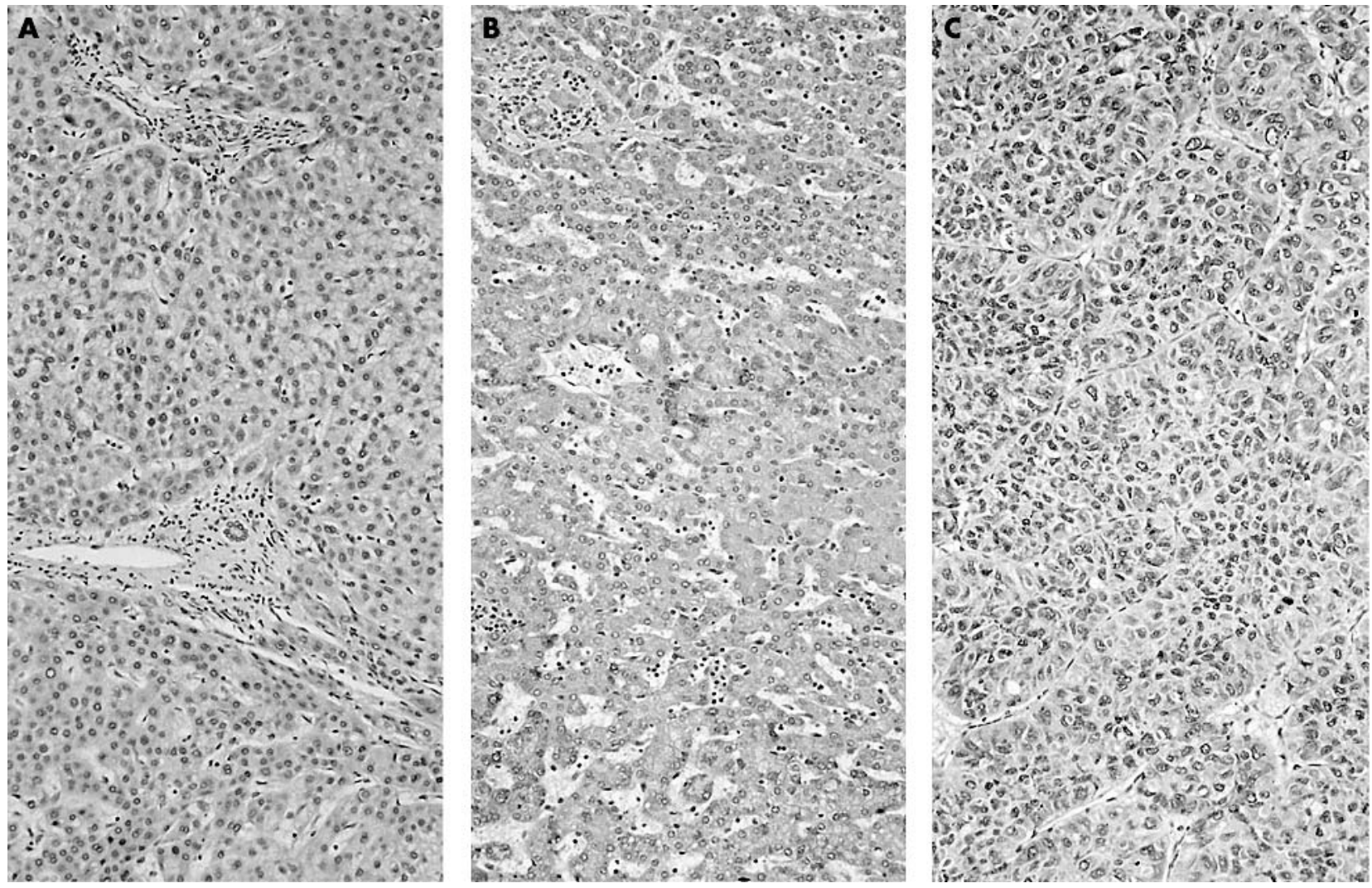

Figure 2 Immunohistochemistry of angiopoietin 2 (Ang-2) in hepatocellular carcinoma (HCC). HCC cells show (A) negative, (B) partially positive, and (C) diffusely positive cytoplasmic staining for Ang-2. Original magnification, $\times 40$.

Immunohistochemically, Tie2 expression was evident in vascular endothelial cells (ECs) and vascular pericytes/ smooth muscle cells (PCs) (fig 4A), and most intense in the periphery of the tumour, particularly in the interface between the tumour and the fibrous capsule, equivalent to Ang-2 expression. The densities of the CD31 positive vessels were higher than those of the Tie 2 positive vessels in all

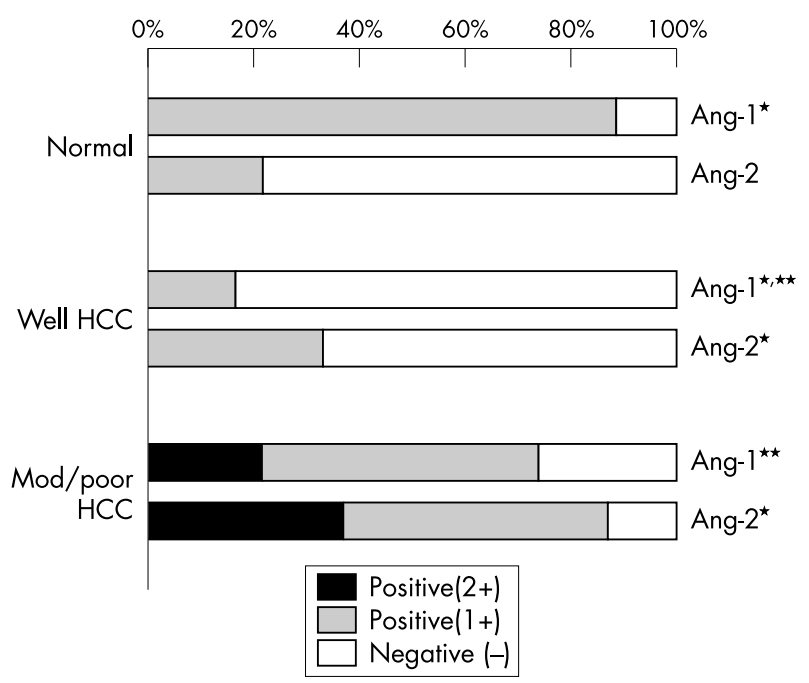

Figure 3 Relation between angiopoietin (Ang) expression and histology of normal liver tissue and hepatocellular carcinoma (HCC). Well HCC, well differentiated hepatocellular carcinoma, mod/poor $\mathrm{HCC}$, moderately and poorly differentiated hepatocellular carcinoma. ${ }^{*} \mathrm{p}<0.01 ;{ }^{* *} \mathrm{p}=0.02$ examined cases (mean (SD) 18.7 (6.8) v 10.6 (4.4)) (fig 4B). Because all the Tie2 positive vessels expressed the CD31 protein, we examined the ratio of Tie 2 : CD31 counts to evaluate Tie2 expression in all 52 HCC cases (mean (SD) ratio, $0.58(0.20))$. Whereas the mean (SD) Tie2: CD31 ratio was $0.40(0.18)$ in well differentiated HCC, it increased to $0.61(0.20)$ in moderately and poorly differentiated HCC. The Tie2: CD31 ratio tended to increase with histological dedifferentiation, and the difference was significant (table 3 ).

\section{RT-PCR analysis of Ang-1, Ang-2, VEGF, and EPO mRNA under hypoxic conditions}

Because Hep3B is a well known human HCC cell line that responds to hypoxia, ${ }^{29}$ we used these cells to determine the effects of hypoxia on the angiogenesis related genes. Semiquantitative RT-PCR analyses showed that the VEGF $615 \mathrm{bp}, 543 \mathrm{bp}$, and $411 \mathrm{bp}$ bands, which correspond to VEGF189, VEGF165, and VEGF121, respectively, were upregulated 3 to 5.5 fold by hypoxia (fig 5). EPO was also upregulated under hypoxic conditions, from 2.5 to 5 fold (fig 5). These data are consistent with the findings in other reports, which used Hep3B and other transformed cultured cells. ${ }^{29}{ }^{31}$ Ang-1 and Ang-2 mRNA were detected in HCC samples, but not in Hep3B cells, under either normoxic or hypoxic conditions (fig 5).

\section{DISCUSSION}

Previous studies have led to the proposal that Ang-1 mediates the stabilisation of developing vessels, ${ }^{32}{ }^{33}$ and that Ang-2 induces vascular destabilisation by blocking Tie2 tyrosine phosphorylation of Ang-1. ${ }^{13}$ Ang-2 plays a facilitative role at sites of tumour vascular remodelling by blocking the constitutive stabilising action of Ang-l in the presence of VEGF. ${ }^{1434} 35$ Earlier, we reported the upregulation and 
Table 2 Comparison of clinicopathological variables dependent on angiopoietin 2 (Ang2) expression

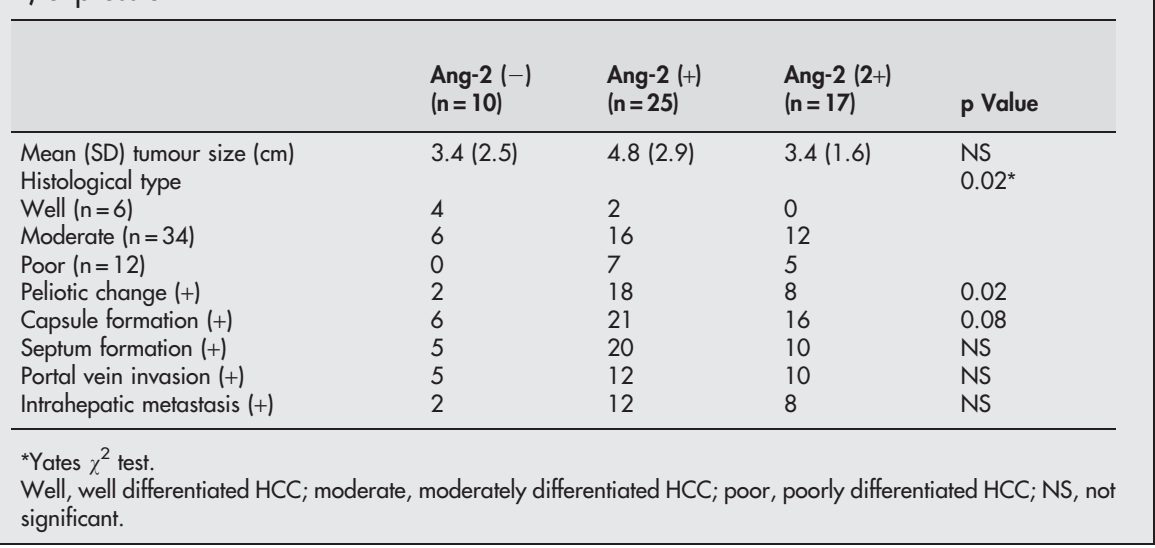
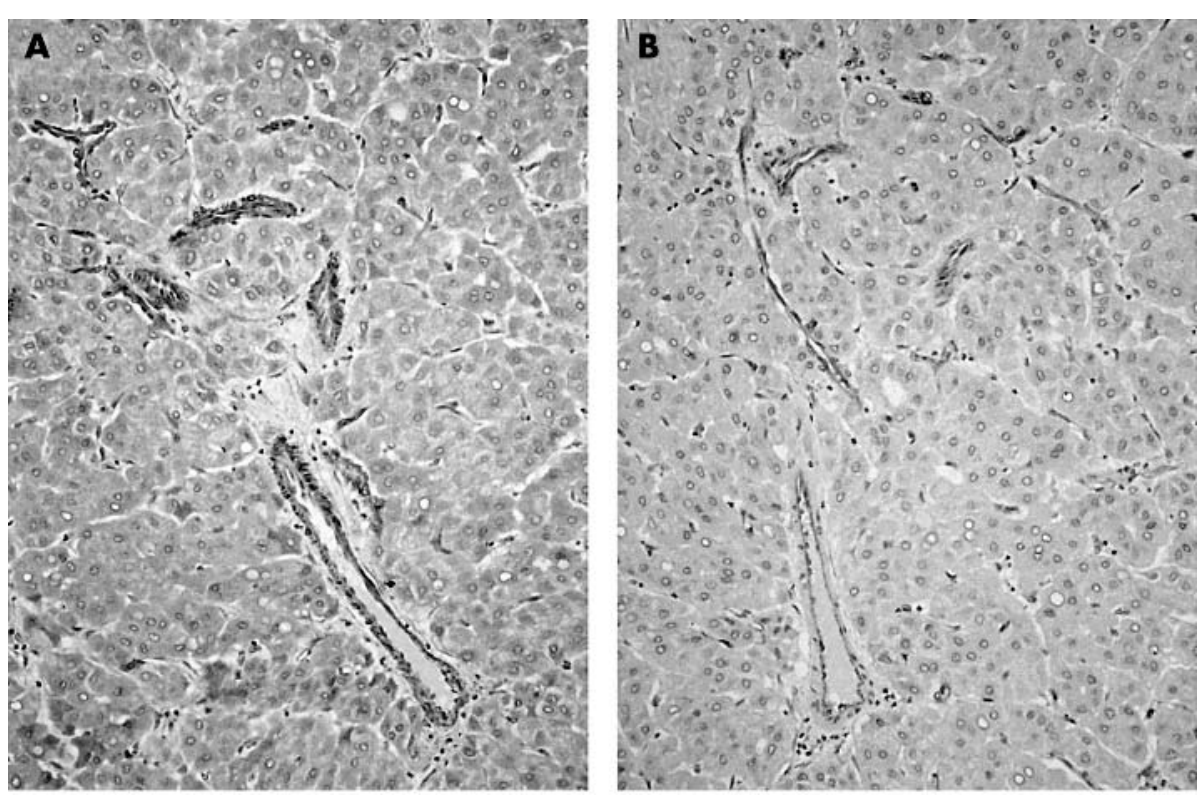

Figure 4 Immunohistochemistry of Tie2 and CD31 in hepatocellular carcinoma (HCC). (A) Both pericytes and endothelial cells of arterial tumour vessels are positive for Tie2; (B) only endothelial cells are CD31 positive in the same sample. Original magnification, $\times 40$. important role of Ang-2 mRNA in hypervascular HCCs, ${ }^{8}$ and the role of Tie2 in HCC progression. ${ }^{20}$ In our present study, we noted strong expression of Ang-2 in human HCC, especially in advanced HCC with hypervascularity, whereas Ang-1 was dominant in non-diseased liver tissue. Ang-2 was strongly expressed at the margin of the tumour and in areas with inflammatory infiltration. It has been reported that VEGF is also expressed in the same areas, and the expression level changes with dedifferentiation in $\mathrm{HCC}^{36}$ suggesting that the
Ang-Tie2 system functions together with VEGF during angiogenesis in HCC.

Interestingly, peliotic change was more frequently seen in Ang-2 positive tumours. Peliotic change, a characteristic feature of HCC, may be associated with the fragility of tumour vessels and congestion of drainage vessels, determined on the basis of histology. ${ }^{30}$ However, the cause of this change remains unclear and the responsible molecules have yet to be defined. In an animal tumour model in which Ang-2

Table 3 Comparison of Tie2 : CD31 microvessel density ratio and histological differentiation

\begin{tabular}{lllll}
\hline & \multicolumn{4}{l}{ Histological differentiation } \\
\cline { 2 - 4 } & Well & Moderate & Poor & Total \\
\hline Tie2 count & $3.1(1.8)$ & $11.6(3.4)$ & $11.4(4.1)$ & $10.6(4.4)$ \\
CD31 count & $8.3(3.0)$ & $20.3(5.7)$ & $19.4(6.8)$ & $18.7(6.8)$ \\
Tie2/CD31 ratio & $0.40(0.18)^{*} \dagger$ & $0.60(0.20)^{*}$ & $0.62(0.19) \dagger$ & $0.58(0.20)$ \\
\hline \multirow{2}{*}{$p=0.02 ; \mathrm{tp}=0.03}$. & & & \\
Values are mean (SD). & & & \\
Well, well differentiated HCC; moderate, moderately differentiated HCC; poor, poorly differentiated HCC; total, \\
average value of all 52 samples.
\end{tabular}


A

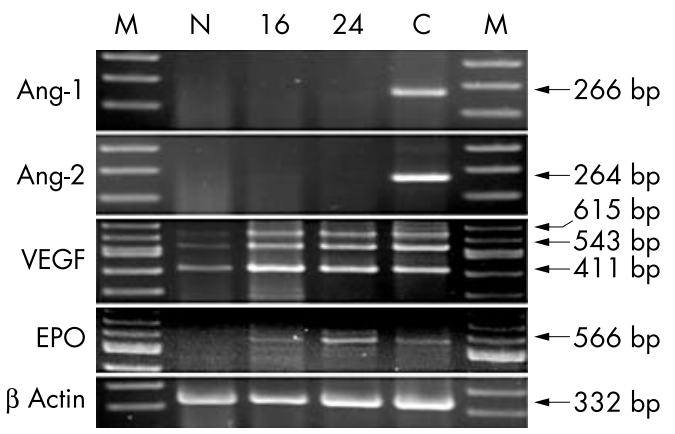

B

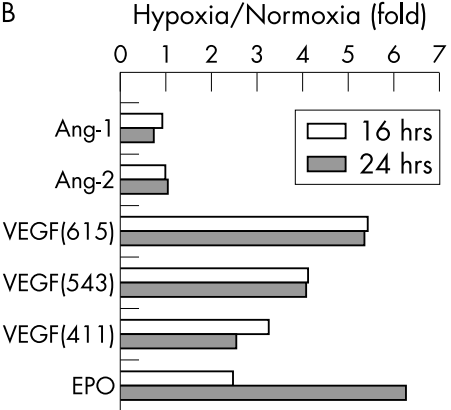

Figure 5 (A) Reverse transcription polymerase chain reaction analysis and (B) semiquantitative analysis of angiopoietin (Ang), vascular endothelial growth factor (VEGF), and erythropoietin (EPO) mRNA in Hep3B cells. Under hypoxic conditions, VEGFs (VEGF189, VEGF165, and VEGF121) and EPO are upregulated approximately 2.5 to 6 fold, but Ang- 1 and Ang-2 mRNA are not detected. $M$, DNA markers; $N$, normoxia; 16,16 hours of hypoxia; 24, 24 hours of hypoxia; C, control hepatocellular carcinoma samples. transfected human HCC cells were injected, significant haemorrhage and peliotic change were observed. ${ }^{8}$ In our present study, we found a significant correlation between Ang-2 expression and peliotic change ( $\mathrm{p}=0.02$; table 1$)$. Our data suggest that destabilisation of tumour vessels induced by Ang-2 may be one cause of peliotic change.

Tie2 expression was particularly apparent at the margin of the tumour and was equivalent to Ang-2 expression. Tie 2 was upregulated in undifferentiated HCCs, but vessels in normal liver tissue and well differentiated HCC also showed some Tie2 expression. Tie2 is upregulated and thought to function as the receptor for Ang-2 at vascular remodelling sites, but is also expressed in normal liver tissue because it is the receptor for both Ang-1 and Ang-2. ${ }^{31}{ }^{37}$ The Tie 2 receptor is pivotal in the angiogenic process because it is involved in the remodelling of the primary vascular plexus into vessels and the recruitment of PCs. ${ }^{20} 32$ In our study, Tie2 expression was not limited to ECs, and expression was also seen on PCs of the tumour vessels in HCC. This suggests that the rapid remodelling of ECs and PCs in the tumour vessels of HCC is regulated by Ang-Tie2 signalling. Ang-2 has been reported to act as an antagonist of Tie2, but recent reports revealed that it also has the potential to phosphorylate Tie2 under some conditions. ${ }^{38}{ }^{39}$ In our study, significant upregulation of Ang2 and Tie 2 was seen, but further studies are required to elucidate the complicated Ang-Tie2 signalling mechanism during the angiogenic processes of HCC.

"The Tie2 receptor is pivotal in the angiogenic process because it is involved in the remodelling of the primary vascular plexus into vessels and the recruitment of vascular pericyte/smooth muscle cells"

The upregulation of Ang-2 has been noted in some human malignant tumours, ${ }^{8} 1516$ but the mechanism was not identified. Tissue hypoxia and the consequent upregulation of hypoxia inducible factor 1 (HIF-1) are known to induce the transcription of VEGF, EPO, and glycolytic enzyme genes in Hep3B HCC cells and other human cancers, ${ }^{29}{ }^{31}$ and the hypoxia-HIF pathway is thought to be a key modulator of angiogenesis. ${ }^{40}$ The effects of hypoxia on Ang-2 expression differ among various cells. In endothelial cells and glioma cells, ${ }^{22-24}$ Ang-2 was reported to be upregulated by hypoxia, whereas Ang-2 was downregulated in renal cell carcinoma cells, ${ }^{41}$ but there are no data for human HCC cells. Thus, we investigated the expression of angiopoietin, VEGF, and EPO mRNA in HCC cells under hypoxic conditions. Our data revealed that the hypoxia-HIF pathway regulates VEGF and EPO expression, but not that of either Ang-1 or Ang-2, in Hep3B HCC cells (fig 5). In fact, the immunohistochemical expression pattern of HIF- $1 \alpha$ was completely different from that of Ang-2 in the HCC tissues (data not shown).
We propose that the stable expression of Ang- 1 is involved in the stabilisation and maturation of vessels in normal liver tissue. Thus, angiopoietin switching-the reversal of Ang-1 and Ang-2 expression-could contribute to vessel wall disassembly in well differentiated HCC. Subsequently, the strong expression of both Ang-2 and Ang-1 at vascular remodelling sites may lead to tumour angiogenesis. Ang-2 expression promotes angiogenesis and may work in concert with VEGF, ${ }^{14}{ }^{35}$ although these two molecules are thought to be regulated through two distinct pathways in HCC.

In summary, we have reported the important role of the Ang-Tie2 system in HCC angiogenesis. The reversal of Ang-1 and Ang-2 expression plays an important role in the angiogenic processes of HCC, being involved in dedifferentiation and progression. Ang-2 might be a potential target for antitumour treatment aimed at blocking the "angiogenic switch" and thereby resulting in tumour dormancy in HCC.

\section{Take home messages}

- Angiopoietin 1 (Ang-1) was more frequently expressed than Ang-2 in the normal liver, whereas Ang- 1 was expressed in $68 \%$ of hepatocellular carcinomas (HCCs) and Ang-2 in $81 \%$, with Ang-2 expression being was significantly higher in poorly differentiated HCCs and in tumours with a peliotic change

- Strong expression of Tie2 was seen in tumour vessels, in accordance with the expression of Ang-2

- In the Hep3B cell line, hypoxic stimuli upregulated vascular endothelial growth factor (VEGF) and erythropoietin, but not Ang-1 or Ang-2.

- Thus, the reversal of Ang-1 and Ang-2 expression seems to play an important role in the angiogenic and dedifferentiation processes in HCC

- Hypoxic stimuli were not responsible for Ang-2 upregulation, unlike that of VEGF, in human HCC cells

\section{ACKNOWLEDGEMENTS}

We thank the staff in the Department of Surgery and Science, Graduate School of Medical Sciences, Kyushu University for providing the tissue materials. We also thank Y Nozuka and $\mathrm{N}$ Tateishi for excellent technical assistance.

\section{Authors' affiliations}

K Sugimachi, K Taguchi, S Aishima, M Tsuneyoshi, Department of Anatomic Pathology, Graduate School of Medical Sciences, Kyushu University, Fukuoka 812-8582, Japan

S Tanaka, M Shimada, K Sugimachi, Department of Surgery and Science, Graduate School of Medical Sciences, Kyushu University, Fukuoka, Japan 


\section{REFERENCES}

1 Hanahan D, Folkman J. Patterns and emerging mechanisms of the angiogenic switch during tumorigenesis. Cell 1996;86:353-64.

2 Folkman J. Angiogenesis in cancer, vascular, rheumatoid and other disease. Nat Med 1995; 1:27-31.

3 Liver cancer study group of Japan. Primary liver cancer in Japan. Clinicopathologic features and results of surgical treatment. Ann Surg 1990;211:277-87.

4 Shimada M, Takenaka K, Gion T, et al. Prognosis of recurrent hepatocellular carcinoma: a 10-year surgical experience in Japan. Gastroenterology 1996:111:720-6.

5 Maeda T, Adachi E, Kajiyama K, et al. CD34 expression in endothelial cells of small hepatocellular carcinoma: its correlation with tumour progression and angiographic findings. J Gastroenterol Hepatol 1995;10:650-4.

6 Sonoda T, Shirabe K, Takenaka K, et al. Angiographically undetected small hepatocellular carcinoma: clinicopathological characteristics, follow-up and treatment. Hepatology 1989;10:1003-7.

7 Sakamoto $M$, Ino Y, Fujii T, et al. Phenotype changes in tumor vessels associated with the progression of hepatocellular carcinoma. Jpn J Clin Oncol 1993;23:98-104.

8 Tanaka S, Mori M, Sakamoto Y, et al. Biologic significance of angiopoietin-2 expression in human hepatocellular carcinoma. J Clin Invest 1999;103:341-5.

9 Davis S, Aldrich TH, Jones PF, et al. Isolation of angiopoietin-1, a ligand for the TIE2 receptor, by secretion-trap expression cloning. Cell 1996:87:1161-9.

10 Maisonpierre PC, Suri C, Jones PF, et al. Angiopoietin-2, a natural antagonist for Tie2 that disrupts in vivo angiogenesis. Science 1997;277:55-60.

11 Partanen J, Armstrong E, Makela TP, et al. A novel endothelial cell surface receptor tyrosine kinase with extracellular epidermal growth factor homology domains. Mol Cell Biol 1992; 12:1698-707.

12 Dumont DJ, Yamaguchi TP, Conlon RA, et al. tek, a novel tyrosine kinase gene located on mouse chromosome 4 , is expressed in endothelial cells and their presumptive precursors. Oncogene 1992;7:1471-80.

13 Sato TN, Qin Y, Kozak CA, et al. Tie-1 and tie-2 define another class of putative receptor tyrosine kinase genes expressed in early embryonic vascular system. Proc Natl Acad Sci U S A 1993;90:9355-8.

14 Holash J, Maisonpierre PC, Compton D, et al. Vessel cooption, regression, and growth in tumors mediated by angiopoietins and VEGF. Science 1999;284:1994-8.

15 Stratmann A, Risau W, Plate KH. Cell type-specific expression of angiopoietin-1 and angiopoietin-2 suggests a role in glioblastoma angiogenesis. Am J Pathol 1998;153:1459-66.

16 Brown LF, Dezube BJ, Tognazzi K, et al. Expression of tie1, tie2, and angiopoietins 1, 2, and 4 in Kaposi's sarcoma and cutaneous angiosarcoma. Am J Pathol 2000;156:2179-83.

17 Chen L, Yang Z, Wang G, et al. Expression of angiopoietin-2 gene and its receptor Tie2 in hepatocellular carcinoma. J Tongii Med Univ 2001 21:228-30, 235

18 Dhar DK, Naora H, Yamanoi A, et al. Requisite role of VEGF receptors in angiogenesis of hepatocellular carcinoma: a comparison with angiopoietin/ Tie pathway. Anticancer Res 2002;22:379-86.

19 Peters KG, Coogan A, Berry D, et al. Expression of Tie2/Tek in breast tumour vasculature provides a new marker for evaluation of tumour angiogenesis. Br J Cancer 1998;77:51-6.

20 Tanaka S, Sugimachi K, Yamashita Yi, et al. Tie2 vascular endothelial receptor expression and function in hepatocellular carcinoma. Hepatology 2002:35:861-7.
21 Kung AL, Wang S, Klco JM, et al. Suppression of tumor growth through disruption of hypoxia-inducible transcription. Nat Med 2000;6:1335-40.

22 Krikun G, Schatz F, Finlay T, et al. Expression of angiopoietin- 2 by human endometrial endothelial cells: regulation by hypoxia and inflammation. Biochem Biophys Res Commun 2000;275:159-63.

23 Oh H, Takagi H, Suzuma K, et al. Hypoxia and vascular endothelial growth factor selectively up-regulate angiopoietin-2 in bovine microvascular endothelial cells. J Biol Chem 1999;274:15732-39.

24 Koga K, Todaka T, Morioka M, et al. Expression of angiopoietin-2 in human glioma cells and its role for angiogenesis. Cancer Res 2001;61:6248-54.

25 Liver Cancer Study Group of Japan. Classification of primary liver cancer, 1 st English ed. Tokyo, Japan: Kanehara, 1997.

26 Ahmad SA, Liu W, Jung YD, et al. Differential expression of angiopoietin-1 and angiopoietin-2 in colon carcinoma. A possible mechanism for the initiation of angiogenesis. Cancer 2001;92:1138-43.

27 Peoch M, Farion R, Hiou A, et al. Immunohistochemical study of VEGF, angiopoietin 2 and their receptors in the neovascularization following microinjection of C6 glioma cells into rat brain. Anticancer Res 2002;22:2147-51

28 Dunk C, Shams M, Niijar S, et al. Angiopoietin-1 and angiopoietin-2 activate trophoblast Tie-2 to promote growth and migration during placental development. Am J Pathol 2000;156:2185-99.

29 Gleadle JM, Ratcliffe PJ. Induction of hypoxia-inducible factor-1, erythropoietin, vascular endothelial growth factor, and glucose transporter-1 by hypoxia: evidence against a regulatory role for Src kinase. Blood 1997;89:503-9.

30 Wanless IR. Vascular disorders. In: MacSween RN, Anthony PP, Scheuer PJ, eds. Pathology of the liver. New York, NY: Churchill Livingstone, 1994:546-8.

31 Ferrara N, Davis-Smyth T. The biology of vascular endothelial growth factor. Endocr Rev 1997;18:4-25.

32 Suri $\mathrm{C}$, Jones PF, Patan $\mathrm{S}$, et al. Requisite role of angiopoietin-1, a ligand for the TIE2 receptor, during embryonic angiogenesis. Cell 1996;87:1171-80.

33 Hanahan D. Signaling vascular morphogenesis and maintenance. Science 1997;277:48-50.

34 Fachinger G, Deutsch U, Risau W. Functional interaction of vascular endothelial-protein-tyrosine phosphatase with the angiopoietin receptor Tie-2. Oncogene 1999;18:5948-53.

35 Holash J, Wiegand SJ, Yancopoulos GD. New model of tumor angiogenesis: dynamic balance between vessel regression and growth mediated by angiopoietins and VEGF. Oncogene 1999;18:5356-62.

36 Yamaguchi R, Yano $H$, lemura A, et al. Expression of vascular endothelial growth factor in human hepatocellular carcinoma. Hepatology 1998:28:68-77.

37 Thurston G, Suri C, Smith K, et al. Leakage-resistant blood vessels in mice transgenically overexpressing angiopoietin-1. Science 1999;286:251 1-14.

$38 \mathrm{Kim} \mathrm{I}, \mathrm{Kim} \mathrm{JH}$, Moon SO, et al. Angiopoietin-2 at high concentration can enhance endothelial cell survival through the phosphatidylinositol 3'-kinase/ Akt signal transduction pathway. Oncogene 2000;19:4549-52.

39 Teichert-Kuliszewska K, Maisonpierre PC, Jones N, et al. Biological action of angiopoietin-2 in a fibrin matrix model of angiogenesis is associated with activation of Tie2. Cardiovasc Res 2001:49:659-70.

40 Semenza GL. HIF-1 and human disease: one highly involved factor. Genes Dev 2000;14:1983-91.

41 Currie MJ, Gunningham SP, Turner K, et al. Expression of the angiopoietins and their receptor Tie2 in human renal clear cell carcinomas; regulation by the von Hippel-Lindau gene and hypoxia. J Pathol 2002;198:502-10. 